\title{
Topological Aspects of Boron Nanotubes
}

\author{
Jia-Bao Liu $\mathbb{D},{ }^{1}$ Hani Shaker $\left(\mathbb{D},{ }^{2}\right.$ Imran Nadeem $\mathbb{D}^{2},{ }^{2}$ and Muhammad Hussain ${ }^{2}$ \\ ${ }^{1}$ School of Mathematics and Physics, Anhui Jianzhu University, Hefei 230601, China \\ ${ }^{2}$ Department of Mathematics, COMSATS University Islamabad, Lahore Campus, Lahore, Pakistan
}

Correspondence should be addressed to Hani Shaker; hani.uet@gmail.com

Received 6 April 2018; Accepted 16 May 2018; Published 4 July 2018

Academic Editor: Jamal Berakdar

Copyright (c) 2018 Jia-Bao Liu et al. This is an open access article distributed under the Creative Commons Attribution License, which permits unrestricted use, distribution, and reproduction in any medium, provided the original work is properly cited.

The degree-based topological indices are used to correlate the physical and chemical properties of a molecule with its chemical structure. Boron nanotubular structures are high-interest materials due to the presence of multicenter bonds and have novel electronic properties. These materials have some important issues in nanodevice applications like mechanical and thermal stability. Therefore, they require theoretical studies on the other properties. In this paper, we present certain degree-based topological indices such as $A B C$, the fourth $A B C, G A$, and the fifth $G A$ indices for boron triangular and boron- $\alpha$ nanotubes.

\section{Introduction}

Mathematical chemistry is a branch of theoretical chemistry in which we get information about the molecular structure by using mathematical techniques without assigning that structure to quantum mechanics $[1,2]$. Chemical graph theory is a branch of mathematical chemistry which implements graph theory to study mathematical modeling of chemical aspects [3]. This theory shows a prominent effect on the extension of the chemical sciences [4].

The chemical structure of a molecule is strongly related to its chemical properties such as strain energy, boiling point, and heat of formation. Molecular graphs can be used to model the chemical structures of molecules and molecular compounds by considering atoms as vertices and the chemical bonds between the atoms as edges. Consider molecular graph $G$ having vertex set $V_{G}$ and edge set $E_{G}$. Let $I_{G}$ be the set of edges of $G$ that are incident with a vertex $p \in V_{p}$, then the degree of $p$ is defined as the cardinality of the set $I_{G}$ and $\delta_{p}=\sum_{q \in N_{p}} d_{q}$, where set $N_{p}$ consists of all neighbor vertices of $p$, that is, $N_{p}=\left\{q \in V_{G} \mid p q \in E_{G}\right\}$.

A topological index is the graph invariant which is used to correlate the physical and chemical properties of a chemical compound with its molecular graph. In this sense, topological indices are based on several topological aspects of the corresponding molecular graph. The use of topological indices is particularly important when using experimental methods leads to waste of time and financial expenditures in large amounts and theoretical methods have not been successful. Topological indices are used to correlate physical properties of chemical structures in QSPR/QSAR studies and provide a measure of structural similarity/stability/diversity of chemical databases. The relative stability of the fullerenes has been correlated with topological indices in [5]. In [6], topological indices are also used to predict the stable isomers of a given fullerene, and for detailed study, we refer [7].

Generally, topological indices can be categorized in three classes: degree-based, distance-based, and spectrum-based indices. In this paper, certain degree-based topological indices are going to be discussed because of their great applications in chemical graph theory. For recent study of distance-based indices, we refer $[8,9]$, and for spectrumbased indices, we refer $[10,11]$.

The first degree-based topological index is the Randić connectivity index which was presented by Randić [12] and is defined as

$$
\chi(G)=\sum_{p q \in E_{G}} \frac{1}{\sqrt{d_{p} d_{q}}} .
$$

This index has been shown to reflect molecular branching and is deeply examined by chemists and mathematicians $[13,14]$. Many physical and chemical properties depend on 


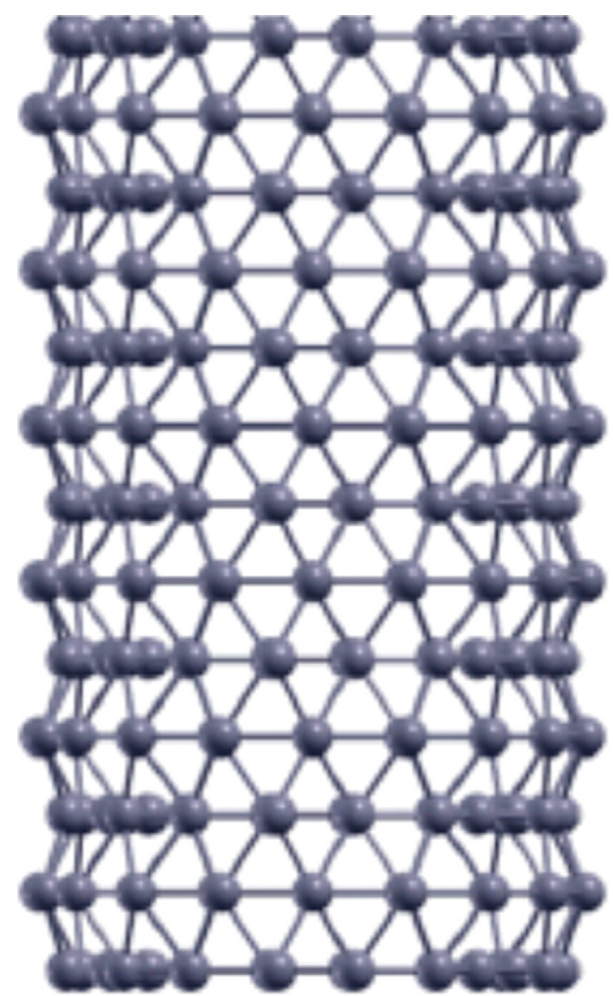

(a)

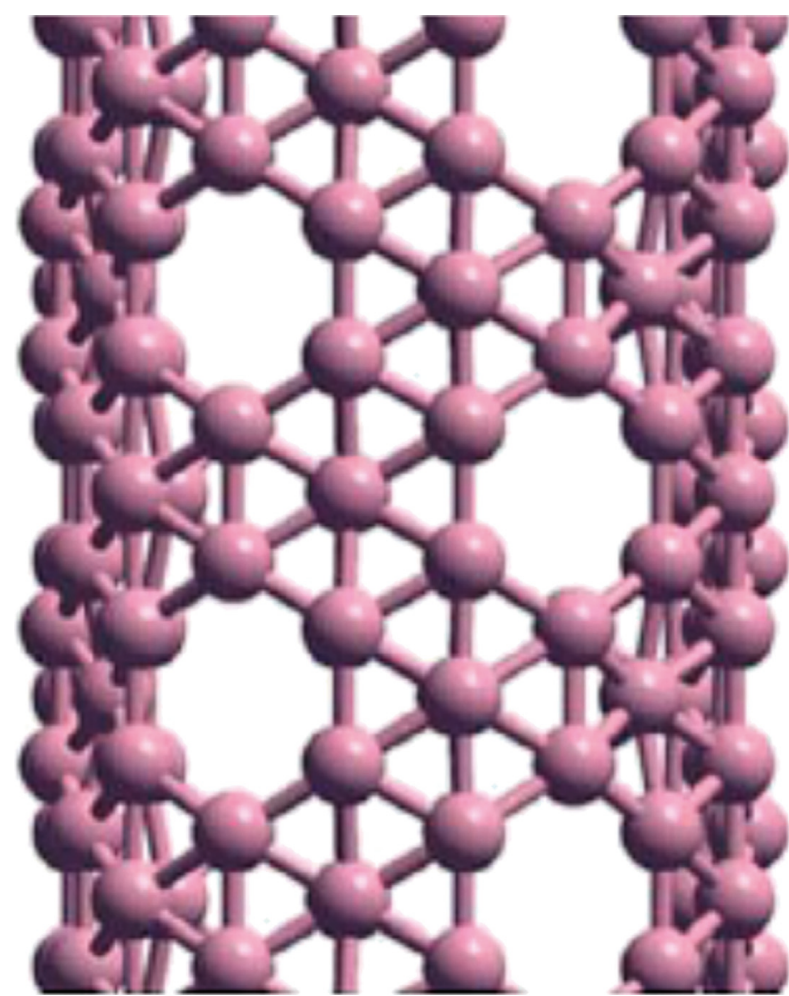

(b)

Figure 1: (a) A 3D perception of boron triangular nanotube. (b) A 3D perception of boron- $\alpha$ nanotube.

such factors which are different rather than branching. With this motivation, Estrada et al. [15] presented the atom-bond connectivity index, which is defined as

$$
A B C(G)=\sum_{p q \in E_{G}} \sqrt{\frac{d_{p}+d_{q}-2}{d_{p} d_{q}}} .
$$

It is reported in [15-17] that this index can be applied in modeling thermodynamic features of organic chemical compounds. In addition, Estrada [16] interpreted a new quantum theory which presented that this index implements a model for consideration of 1,2-, 1,3-, and 1,4-interactions in the carbon-atom skeleton of saturated hydrocarbons, and in this sense, it can be applied for rationalizing steric effects in such compounds.

Recently, Ghorbani and Hosseinzadeh [18] presented the fourth atom-bond connectivity index which is defined as

$$
A B C_{4}(G)=\sum_{p q \in E_{G}} \sqrt{\frac{\delta_{p}+\delta_{q}-2}{\delta_{p} \delta_{q}}} .
$$

The sense for presenting a new index is to increase prediction of some property of molecules. One of the successors of the Randić connectivity index called the geometric-arithmetic (GA) connectivity index which is presented by Vukičević and Furtula [19] is defined as

$$
G A(G)=\sum_{p q \in E_{G}} \frac{2 \sqrt{d_{p} d_{q}}}{\left(d_{p}+d_{q}\right)} .
$$

It is reported in $[19,20]$ that this index provides better prediction rate than the Randić index in various physicochemical properties such as entropy, enthalpy of formation, and standard enthalpy of vaporization. Furthermore, the enhancement in the prediction accuracy rate of the GA index comparing to the Randic index is more than 9\% for the standard enthalpy of vaporization. In [21], it also presents that there forms excellent correlation between heat of formation of benzenoid hydrocarbons and the GA index (the correlation coefficient is 0.972).

Recently, the fifth GA index is presented by Graovac et al. [22] and is defined as

$$
G A_{5}(G)=\sum_{p q \in E_{G}} \frac{2 \sqrt{\delta_{p} \delta_{q}}}{\left(\delta_{p}+\delta_{q}\right)} .
$$

In this paper, we present $A B C, A B C_{4}, G A$, and $G A_{5}$ topological indices for new classes of nanotubes fabricated from carbon hexagonal nanotubes called the boron triangular and boron $\alpha$ nanotubes.

\section{Boron Nanotubes}

In last 20 years, various types of boron-containing nanomaterials such as boron nanoclusters, boron nanowires, boron nanotubes, boron nanobelts, boron nanoribbons, boron nanosheets, and boron fullerenes have been experimentally synthesized and identified. Boron nanomaterials have been considered as excellent materials for enhancing the characteristics of optoelectronic nanodevices because of their broad elastic modulus, high-melting 


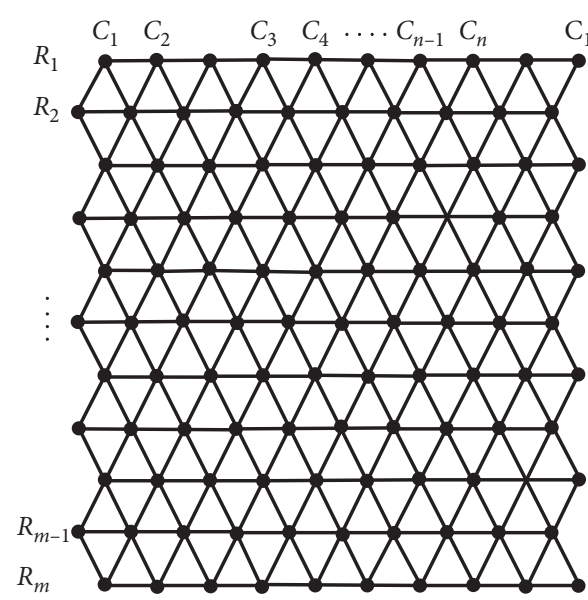

(a)

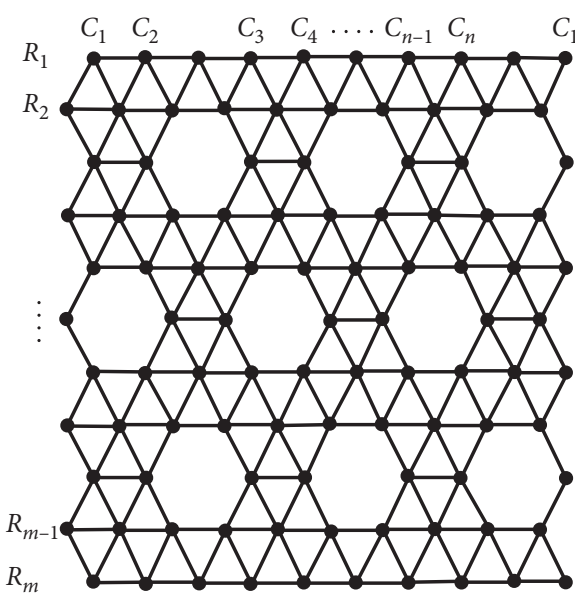

(b)

FIgURe 2: (a) A 2D sheet of boron triangular nanotube BT $[m, n]$. (b) A 2D sheet of boron- $\alpha$ nanotube BA $[m, n]$.

point, excessive conductivity, great emission uniformity, and low turn-on field. These materials can carry excessive emission current, which recommends that they may have great prospective applications in the field emission area [23]. Furthermore, boron nanomaterials also have some better properties compared to carbon nanomaterials such as excessive resistance to oxidation at high temperatures and great chemical stability and are stable broad band-gap semiconductor [24,25]. Due to these properties, boron nanomaterials may have great applications at high temperatures or in corrosive environments functioning as supercapacitors, solid lubricants, fuel cells, and batteries [26]. Moreover, the extensive range of boron nanomaterials themselves could be the building blocks for combining with other existing nanomaterials to design and create materials with new properties. For this reason, the boron-nitrogen clusters have been developed in [27, 28], and boron-nitrogen cages have been computed in [29]. The boron fullerenes are the cage molecules [30] which can serve as the building blocks for fabrication of new hybrid nanostructures with novel properties.

The boron triangular nanotube was created in 2004 [23] and obtained from a carbon hexagonal nanotube by adding an extra atom to the center of each hexagon. Also, a special boron nanotube was fabricated from a carbon hexagonal nanotube in 2008, by adding an extra atom to the center of certain hexagons $[31,32]$. This nanotube is designed by generating a mixture of hexagons and triangles called the boron- $\alpha$ nanotube. It is reported in $[33,34]$ that these nanotubes are important materials for optical, electronic, chemical, and biosensing applications. The comparison study about some computational aspects of boron triangular and boron- $\alpha$ nanotubes has been investigated in [35]. The 3D perceptions of boron triangular and boron- $\alpha$ nanotube are presented in Figure 1.

\section{Main Results}

We denote the molecular graphs of boron triangular and boron- $\alpha$ nanotubes by BT $[m, n]$ and BA $[m, n]$, respectively, where $m$ is the number of rows and $n$ is the number of columns in a $2 \mathrm{D}$ sheet of $\mathrm{BT}[m, n]$ or $\mathrm{BA}[m, n]$ as shown in
TABLE 1: The order and size of boron nanotubes.

\begin{tabular}{lcc}
\hline Molecular graph & Order & Size \\
\hline $\mathrm{BT}[m, n]$ & $3 m n / 2$ & $3 n(3 m-2) / 2$ \\
$\mathrm{BA}(X)[m, n]$ & $n(4 m+1) / 3$ & $n(7 m-2) / 2$ \\
$\mathrm{BA}(Y)[m, n]$ & $4 m n / 3$ & $n(7 m-4) / 2$ \\
\hline
\end{tabular}

Figure 2. We categorize the boron- $\alpha$ nanotubes into two classes with respect to $m$. We denote these classes as $\mathrm{BA}(X)[m, n]$ and $\mathrm{BA}(Y)[m, n]$ for $m \equiv 2 \bmod 3$ and $m \equiv 0$ $\bmod 3$, respectively. The order and size of $\mathrm{BT}[m, n]$, $\mathrm{BA}(X)[m, n]$, and $\mathrm{BA}(Y)[m, n]$ are given in Table 1 . In the following theorem, we compute the $A B C$ index, fourth $A B C$, $G A$, and fifth $G A$ indices for $\mathrm{BT}[m, n]$.

Theorem 1. Consider the boron triangular nanotube $B T[m, n]$, where $m \geq 3$ and $n$ even, then

$$
\begin{aligned}
A B C(\mathrm{BT}[m, n])= & \frac{3 \sqrt{10}}{4} m n+\frac{3 \sqrt{6}}{4} n-2 \sqrt{10} n+2 \sqrt{3} n, \\
G A(\operatorname{BT}[m, n])= & \frac{9}{2} m n+\frac{12 \sqrt{6}}{5} n-9 n, \\
A B C_{4}(\operatorname{BT}[m, n])= & \frac{\sqrt{70}}{8} m n-\frac{7 \sqrt{70}}{12} n+\frac{3 \sqrt{62}}{32} n \\
& +\frac{3 \sqrt{38}}{20} n+\frac{\sqrt{33}}{4} n+\frac{3 \sqrt{5}}{4} n, \\
G A_{5}(\operatorname{BT}[m, n])= & \frac{9}{2} m n+\frac{72 \sqrt{2}}{17} n+\frac{24 \sqrt{10}}{13} n-15 n .
\end{aligned}
$$

Proof. Consider the boron triangular nanotube $G=\mathrm{BT}[m, n]$. There are three partite subsets of the edge set $E_{G}$ corresponding to the degree of end vertices which are presented as 


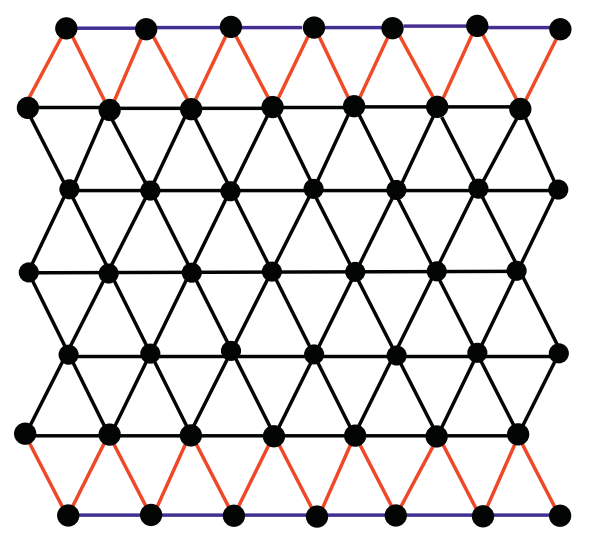

(a)

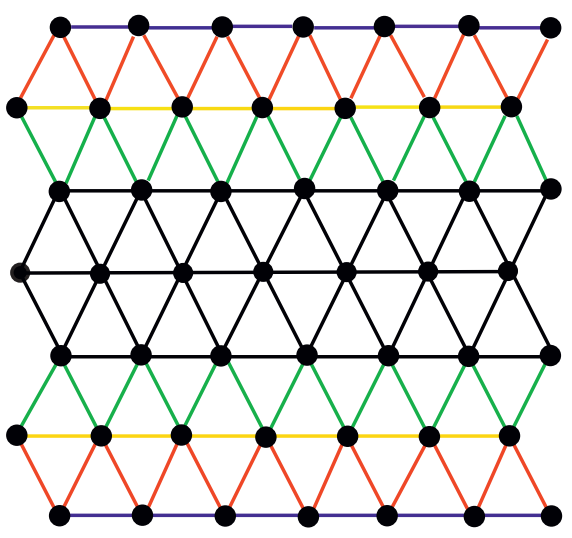

(b)

FIGURE 3: The edge partitions of BT $[7,4]$ nanotube: (a) with respect to degree of end vertices; (b) with respect to degree sum of neighbor vertices.

$$
\begin{aligned}
& E_{4,4}=\left\{p q \in E_{G} \mid d_{p}=d_{q}=4\right\}, \\
& E_{4,6}=\left\{p q \in E_{G} \mid d_{p}=4 \text { and } d_{q}=6\right\}, \\
& E_{6,6}=\left\{p q \in E_{G} \mid d_{p}=d_{q}=6\right\} .
\end{aligned}
$$

Therefore, we have $\left|E_{4,4}\right|=3 n,\left|E_{4,6}\right|=6 n$, and $\left|E_{6,6}\right|=$ $3 n(3 m-8) / 2$. The representative edges of these partite sets are shown in Figure 3(a) in which blue, red, and black edges belong to $E_{4,4}, E_{4,6}$, and $E_{6,6}$, respectively. From (2) and (4), the $A B C$ and $G A$ indices of $G$ are formulated as

$$
\begin{aligned}
A B C(G)= & \sum_{p q \in E_{4,4}} \sqrt{\frac{d_{p}+d_{q}-2}{d_{p} d_{q}}}+\sum_{p q \in E_{4,6}} \sqrt{\frac{d_{p}+d_{q}-2}{d_{p} d_{q}}} \\
& +\sum_{p q \in E_{6,6}} \sqrt{\frac{d_{p}+d_{q}-2}{d_{p} d_{q}}} \\
= & \frac{3 \sqrt{10}}{4} m n+\frac{3 \sqrt{6}}{4} n-2 \sqrt{10} n+2 \sqrt{3} n, \\
G A(G)= & \sum_{p q \in E_{4,4}} \frac{2 \sqrt{d_{p} d_{q}}}{d_{p}+d_{q}}+\sum_{p q \in E_{4,6}} \frac{2 \sqrt{d_{p} d_{q}}}{d_{p}+d_{q}}+\sum_{p q \in E_{6,6}} \frac{2 \sqrt{d_{p} d_{q}}}{d_{p}+d_{q}} \\
= & \frac{9}{2} m n+\frac{12 \sqrt{6}}{5} n-9 n .
\end{aligned}
$$

Similarly, the edge partite subsets of $E_{G}$ corresponding to their degree sum of neighbors of end vertices are given as

$$
\begin{aligned}
& S_{20,20}=\left\{p q \in E_{G} \mid \delta_{p}=\delta_{q}=20\right\}, \\
& S_{20,32}=\left\{p q \in E_{G} \mid \delta_{p}=20 \text { and } \delta_{q}=32\right\}, \\
& S_{32,32}=\left\{p q \in E_{G} \mid \delta_{p}=\delta_{q}=32\right\}, \\
& S_{32,36}=\left\{p q \in E_{G} \mid \delta_{p}=32 \text { and } \delta_{q}=36\right\}, \\
& S_{36,36}=\left\{p q \in E_{G} \mid \delta_{p}=\delta_{q}=36\right\} .
\end{aligned}
$$

Therefore, we have $\left|S_{20,20}\right|=3 q,\left|S_{20,32}\right|=6 q,\left|S_{32,32}\right|=3 q$, $\left|S_{32,36}\right|=6 q$, and $\left|S_{36,36}\right|=3 q(3 p-14) / 2$. The representatives of these partite sets are shown in Figure 3(b) in which blue, red, yellow, green, and black edges belong to $S_{20,20}$, $S_{20,32}, S_{32,32}, S_{32,36}$, and $S_{36,36}$, respectively. From (3) and (5), the fourth $A B C$ and fifth $G A$ indices of $G$ are computed as

$$
\begin{aligned}
& A B C_{4}(G)=\sum_{p q \in S_{20,20}} \sqrt{\frac{\delta_{p}+\delta_{q}-2}{\delta_{p} \delta_{q}}}+\sum_{p q \in S_{20,32}} \sqrt{\frac{\delta_{p}+\delta_{q}-2}{\delta_{p} \delta_{q}}} \\
& +\sum_{p q \in S_{32,32}} \sqrt{\frac{\delta_{p}+\delta_{q}-2}{\delta_{p} \delta_{q}}}+\sum_{p q \in S_{32,36}} \sqrt{\frac{\delta_{p}+\delta_{q}-2}{\delta_{p} \delta_{q}}} \\
& +\sum_{p q \in S_{36,36}} \sqrt{\frac{\delta_{p}+\delta_{q}-2}{\delta_{p} \delta_{q}}} \\
& =\frac{\sqrt{70}}{8} m n-\frac{7 \sqrt{70}}{12} n+\frac{3 \sqrt{62}}{32} n \\
& +\frac{3 \sqrt{38}}{20} n+\frac{\sqrt{33}}{4} n+\frac{3 \sqrt{5}}{4} n, \\
& G A_{5}(G)=\sum_{p q \in S_{20,20}} \frac{2 \sqrt{\delta_{p} \delta_{q}}}{\delta_{p}+\delta_{q}}+\sum_{p q \in S_{20,32}} \frac{2 \sqrt{\delta_{p} \delta_{q}}}{\delta_{p}+\delta_{q}}+\sum_{p q \in S_{32,32}} \frac{2 \sqrt{\delta_{p} \delta_{q}}}{\delta_{p}+\delta_{q}} \\
& +\sum_{p q \in S_{32,36}} \frac{2 \sqrt{\delta_{p} \delta_{q}}}{\delta_{p}+\delta_{q}}+\sum_{p q \in S_{36,36}} \frac{2 \sqrt{\delta_{p} \delta_{q}}}{\delta_{p}+\delta_{q}} \\
& =\frac{9}{2} m n+\frac{72 \sqrt{2}}{17} n+\frac{24 \sqrt{10}}{13} n-15 n .
\end{aligned}
$$

In next theorem, we formulate $A B C$, the fourth $A B C, G A$, and the fifth $G A$ indices for $\operatorname{BA}(X)[m, n]$ nanotubes. 
Theorem 2. Consider the boron- $\alpha$ nanotube $B A(X)[m, n]$, then

$$
\begin{aligned}
& A B C(\mathrm{BA}(X)[m, n])=\frac{3 \sqrt{2}}{5} m n+\frac{6}{\sqrt{30}} m n+\frac{3 \sqrt{6}}{4} n \\
& +\frac{4 \sqrt{7}}{\sqrt{20}} n+\frac{2}{\sqrt{3}} n-\frac{8 \sqrt{2}}{5} n-\frac{18}{\sqrt{30}} n \\
& G A(\mathrm{BA}(X)[m, n])=\frac{4 \sqrt{30}}{11} m n+\frac{3}{2} m n+\frac{16 \sqrt{5}}{9} n \\
& +\frac{8 \sqrt{6}}{10} n-\frac{12 \sqrt{30}}{11} n-n, \\
& A B C_{4}(\mathrm{BA}(X)[m, n])=\frac{\sqrt{13}}{9} m n+\frac{\sqrt{22}}{9} m n+\frac{2 \sqrt{35}}{3 \sqrt{38}} n \\
& +\frac{\sqrt{10}}{3 \sqrt{3}} n+\frac{\sqrt{41}}{6} n+\frac{3 \sqrt{5}}{\sqrt{133}} n \\
& +\frac{7}{9 \sqrt{2}} n+\frac{5}{\sqrt{84}} n+\frac{6}{19} n+\frac{\sqrt{46}}{24} n \\
& +\frac{\sqrt{53}}{3 \sqrt{21}} n-\frac{14 \sqrt{13}}{27} n-\frac{5 \sqrt{22}}{9} n \\
& G A_{5}(\mathrm{BA}(X)[m, n])=\frac{36 \sqrt{10}}{57} m n+\frac{3}{2} m n+\frac{12 \sqrt{38}}{37} n \\
& +\frac{8 \sqrt{3}}{7} n+\frac{8 \sqrt{114}}{43} n+\frac{8 \sqrt{133}}{47} n \\
& +\frac{24 \sqrt{2}}{17} n+\frac{4 \sqrt{42}}{13} n \\
& +\frac{24 \sqrt{21}}{55} n-\frac{180 \sqrt{10}}{57} n-5 n \text {. }
\end{aligned}
$$

Proof. Consider the boron- $\alpha$ nanotube $H=\mathrm{BA}(X)[m, n]$. There are five partite subsets of the edge set $E_{H}$ corresponding to the degree of end vertices which are given as

$$
\begin{aligned}
& E_{4,4}=\left\{p q \in E_{H} \mid d_{p}=d_{q}=4\right\}, \\
& E_{4,5}=\left\{p q \in E_{H} \mid d_{p}=4 \text { and } d_{q}=5\right\}, \\
& E_{4,6}=\left\{p q \in E_{H} \mid d_{p}=4 \text { and } d_{q}=6\right\}, \\
& E_{5,5}=\left\{p q \in E_{H} \mid d_{p}=d_{q}=5\right\}, \\
& E_{5,6}=\left\{p q \in E_{H} \mid d_{p}=5 \text { and } d_{q}=6\right\} .
\end{aligned}
$$

Therefore, we have $\left|E_{4,4}\right|=3 n,\left|E_{4,5}\right|=4 n,\left|E_{4,6}\right|=$ $2 n, \quad\left|E_{5,5}\right|=n(3 m-8) / 2$, and $\left|E_{5,6}\right|=2 n(m-3)$. The

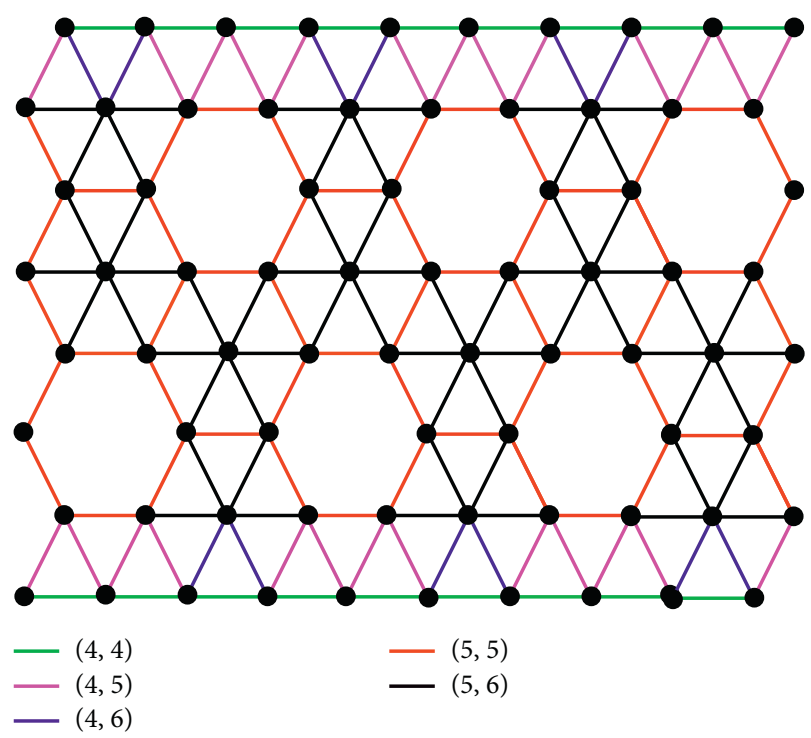

FIgURE 4: The edge partitions of $\mathrm{BA}(X)[8,6]$ with respect to degree of end vertices.

representative edges of these partite sets are presented in Figure 4. From (2) and (4), the $A B C$ and $G A$ indices of $H$ are formulated as

$$
\begin{aligned}
& A B C(H)= \sum_{p q \in E_{4,4}} \sqrt{\frac{d_{p}+d_{q}-2}{d_{p} d_{q}}}+\sum_{p q \in E_{4,5}} \sqrt{\frac{d_{p}+d_{q}-2}{d_{p} d_{q}}} \\
&+\sum_{p q \in E_{4,6}} \sqrt{\frac{d_{p}+d_{q}-2}{d_{p} d_{q}}}+\sum_{p q \in E_{5,5}} \sqrt{\frac{d_{p}+d_{q}-2}{d_{p} d_{q}}} \\
&+\sum_{p q \in E_{5,6}} \sqrt{\frac{d_{p}+d_{q}-2}{d_{p} d_{q}}} \\
&= \frac{3 \sqrt{2}}{5} m n+\frac{6}{\sqrt{30}} m_{n}+\frac{3 \sqrt{6}}{4} n+\frac{4 \sqrt{7}}{\sqrt{20} n} \\
&+\sum_{p q \in E_{5,6}} \frac{2}{\sqrt{3}} n-\frac{8 \sqrt{2}}{5} n-\frac{18}{\sqrt{30}} n, \\
&+\sum_{p q \in E_{4,6}} \frac{2 \sqrt{d_{p} d_{q}}}{d_{p}+d_{q}}+\sum_{p q \in E_{5,5}} \frac{2 \sqrt{d_{p} d_{q}}}{d_{p} d_{q}} \\
& \sum_{p q \in(H)} \frac{2 \sqrt{d_{p} d_{q}}}{d_{p}+d_{q}}+\sum_{p q \in E_{4,5}} \frac{2 \sqrt{d_{p} d_{q}}}{d_{p}+d_{q}}
\end{aligned}
$$




$$
\begin{aligned}
= & \frac{4 \sqrt{30}}{11} m n+\frac{3}{2} m n+\frac{16 \sqrt{5}}{9} n \\
& +\frac{8 \sqrt{6}}{10} n-\frac{12 \sqrt{30}}{11} n-n .
\end{aligned}
$$

Also, there are eleven edge partite subsets of $E_{G}$ corresponding to their degree sum of neighbors of end vertices, which are given as

$$
\begin{aligned}
& S_{18,19}=\left\{p q \in E_{H} \mid \delta_{p}=18 \text { and } \delta_{q}=19\right\}, \\
& S_{18,24}=\left\{p q \in E_{H} \mid \delta_{p}=18 \text { and } \delta_{q}=24\right\}, \\
& S_{19,19}=\left\{p q \in E_{H} \mid \delta_{p}=\delta_{q}=19\right\}, \\
& S_{19,24}=\left\{p q \in E_{H} \mid \delta_{p}=19 \text { and } \delta_{q}=24\right\}, \\
& S_{19,28}=\left\{p q \in E_{H} \mid \delta_{p}=19 \text { and } \delta_{q}=28\right\}, \\
& S_{24,24}=\left\{p q \in E_{H} \mid \delta_{p}=\delta_{q}=24\right\}, \\
& S_{24,27}=\left\{p q \in E_{H} \mid \delta_{p}=24 \text { and } \delta_{q}=27\right\}, \\
& S_{24,28}=\left\{p q \in E_{H} \mid \delta_{p}=24 \text { and } \delta_{q}=28\right\}, \\
& S_{27,27}=\left\{p q \in E_{H} \mid \delta_{p}=\delta_{q}=27\right\}, \\
& S_{27,28}=\left\{p q \in E_{H} \mid \delta_{p}=27 \text { and } \delta_{q}=28\right\}, \\
& S_{27,30}=\left\{p q \in E_{H} \mid \delta_{p}=27 \text { and } \delta_{q}=30\right\} .
\end{aligned}
$$

Therefore, we have $\left|S_{18,19}\right|=2 n,\left|S_{18,24}\right|=2 n,\left|S_{19,19}\right|=n$, $\left|S_{19,24}\right|=2 n,\left|S_{19,28}\right|=2 n,\left|S_{24,24}\right|=n,\left|S_{24,27}\right|=2 n,\left|S_{24,28}\right|=$ $2 n, \quad\left|S_{27,27}\right|=n(3 m-14) / 2, \quad\left|S_{27,28}\right|=2 n$, and $\left|S_{27,30}\right|=$ $2 n(m-5)$. The representative edges of these partite sets are presented in Figure 5. From (3) and (5), the fourth $A B C$ index and fifth $G A$ index are computed as

$$
\begin{aligned}
A B C_{4}(G)= & \sum_{p q \in S_{18,19}} \sqrt{\frac{\delta_{p}+\delta_{q}-2}{\delta_{p} \delta_{q}}}+\sum_{p q \in S_{18,24}} \sqrt{\frac{\delta_{p}+\delta_{q}-2}{\delta_{p} \delta_{q}}} \\
& +\sum_{p q \in S_{19,19}} \sqrt{\frac{\delta_{p}+\delta_{q}-2}{\delta_{p} \delta_{q}}}+\sum_{p q \in S_{19,24}} \sqrt{\frac{\delta_{p}+\delta_{q}-2}{\delta_{p} \delta_{q}}} \\
& +\sum_{p q \in S_{19,28}} \sqrt{\frac{\delta_{p}+\delta_{q}-2}{\delta_{p} \delta_{q}}}+\sum_{p q \in S_{24,24}} \sqrt{\frac{\delta_{p}+\delta_{q}-2}{\delta_{p} \delta_{q}}} \\
& +\sum_{p q \in S_{24,27}} \sqrt{\frac{\delta_{p}+\delta_{q}-2}{\delta_{p} \delta_{q}}}+\sum_{p q \in S_{24,28}} \sqrt{\frac{\delta_{p}+\delta_{q}-2}{\delta_{p} \delta_{q}}}
\end{aligned}
$$

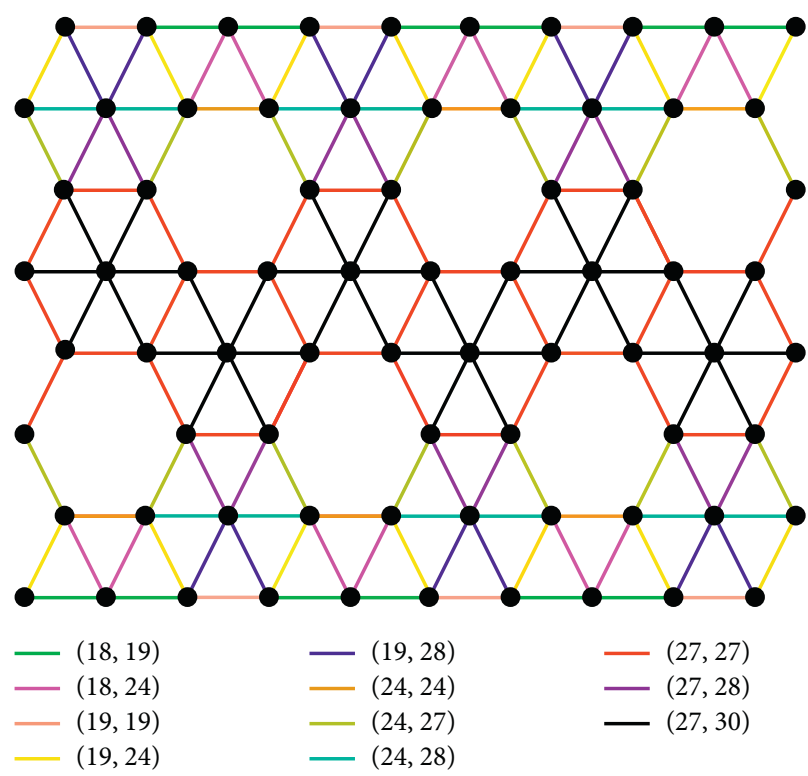

FIgURE 5: The edge partitions of $\mathrm{BA}(X)[8,6]$ with respect to degree sum of neighbor vertices.

$$
\begin{aligned}
& +\sum_{p q \in S_{27,27}} \sqrt{\frac{\delta_{p}+\delta_{q}-2}{\delta_{p} \delta_{q}}}+\sum_{p q \in S_{27,28}} \sqrt{\frac{\delta_{p}+\delta_{q}-2}{\delta_{p} \delta_{q}}} \\
& +\sum_{p q \in S_{27,30}} \sqrt{\frac{\delta_{p}+\delta_{q}-2}{\delta_{p} \delta_{q}}} \\
& =\frac{\sqrt{13}}{9} m n+\frac{\sqrt{22}}{9} m n+\frac{2 \sqrt{35}}{3 \sqrt{38}} n+\frac{\sqrt{10}}{3 \sqrt{3}} n \\
& +\frac{\sqrt{41}}{6} n+\frac{3 \sqrt{5}}{\sqrt{133}} n+\frac{7}{9 \sqrt{2}} n+\frac{5}{\sqrt{84}} n \\
& +\frac{6}{19} n+\frac{\sqrt{46}}{24} n+\frac{\sqrt{53}}{3 \sqrt{21}} n-\frac{14 \sqrt{13}}{27} n-\frac{5 \sqrt{22}}{9} n, \\
& G A_{5}(G)=\sum_{p q \in S_{18,19}} \frac{2 \sqrt{\delta_{p} \delta_{q}}}{\delta_{p}+\delta_{q}}+\sum_{p q \in S_{18,24}} \frac{2 \sqrt{\delta_{p} \delta_{q}}}{\delta_{p}+\delta_{q}} \\
& +\sum_{p q \in S_{19,19}} \frac{2 \sqrt{\delta_{p} \delta_{q}}}{\delta_{p}+\delta_{q}}+\sum_{p q \in S_{19,24}} \frac{2 \sqrt{\delta_{p} \delta_{q}}}{\delta_{p}+\delta_{q}} \\
& +\sum_{p q \in S_{19,28}} \frac{2 \sqrt{\delta_{p} \delta_{q}}}{\delta_{p}+\delta_{q}}+\sum_{p q \in S_{24,24}} \frac{2 \sqrt{\delta_{p} \delta_{q}}}{\delta_{p}+\delta_{q}} \\
& +\sum_{p q \in S_{24,27}} \frac{2 \sqrt{\delta_{p} \delta_{q}}}{\delta_{p}+\delta_{q}}+\sum_{p q \in S_{24,28}} \frac{2 \sqrt{\delta_{p} \delta_{q}}}{\delta_{p}+\delta_{q}}
\end{aligned}
$$




$$
\begin{aligned}
& +\sum_{p q \in S_{27,27}} \frac{2 \sqrt{\delta_{p} \delta_{q}}}{\delta_{p}+\delta_{q}}+\sum_{p q \in S_{27,28}} \frac{2 \sqrt{\delta_{p} \delta_{q}}}{\delta_{p}+\delta_{q}} \\
& +\sum_{p q \in S_{27,30}} \frac{2 \sqrt{\delta_{p} \delta_{q}}}{\delta_{p}+\delta_{q}} \\
& =\frac{36 \sqrt{10}}{57} m n+\frac{3}{2} m n+\frac{12 \sqrt{38}}{37} n+\frac{8 \sqrt{3}}{7} n+\frac{8 \sqrt{114}}{43} n \\
& +\frac{8 \sqrt{133}}{47} n+\frac{24 \sqrt{2}}{17} n+\frac{4 \sqrt{42}}{13} n \\
& +\frac{24 \sqrt{21}}{55} n-\frac{180 \sqrt{10}}{57} n-5 n .
\end{aligned}
$$

For BA $(Y)[m, n]$ nanotube, $A B C$, the fourth $A B C, G A$ and the fifth $G A$ indices are formulated in Theorem 3.

Theorem 3. Consider the boron- $\alpha$ nanotube $B A(Y)[m, n]$, then

$$
\begin{aligned}
A B C(\mathrm{BA}(Y)[m, n])= & \frac{6}{\sqrt{50}} m n+\frac{\sqrt{30}}{5} m n+\frac{3 \sqrt{6}}{8} n \\
& +\sqrt{\frac{2}{5}} n+\frac{\sqrt{7}}{3 \sqrt{2}} n+\frac{\sqrt{7}}{\sqrt{5}} n+\frac{1}{\sqrt{3}} n \\
& +\frac{1}{3} n-\frac{8 \sqrt{2}}{5} n-\frac{\sqrt{30}}{2} n, \\
G A(\mathrm{BA}(Y)[m, n])= & \frac{4 \sqrt{30}}{11} m n+\frac{3}{2} m n+\frac{\sqrt{15}}{4} n \\
& +\frac{2 \sqrt{2}}{3} n+\frac{8 \sqrt{5}}{9} n \\
& +\frac{2 \sqrt{6}}{5} n-\frac{10 \sqrt{30}}{11} n-2 n, \\
A B C_{4}(\mathrm{BA}(Y)[m, n])= & \frac{\sqrt{13}}{9} m n+\frac{2 \sqrt{5}}{9} m n+\frac{\sqrt{26}}{28} n \\
& +\frac{\sqrt{37}}{5 \sqrt{14}} n+\frac{\sqrt{38}}{2 \sqrt{91}} n+\frac{\sqrt{35}}{3 \sqrt{38}} n \\
& +\frac{\sqrt{10}}{6 \sqrt{3}} n+\frac{3}{19} n+\frac{\sqrt{41}}{2 \sqrt{114}} n \\
& +\frac{3 \sqrt{5}}{2 \sqrt{133}} n+\frac{\sqrt{46}}{48} n+\frac{7}{18 \sqrt{2}} n \\
& +\frac{5}{4 \sqrt{21}} n+\frac{2 \sqrt{3}}{25} n+\frac{7}{5 \sqrt{26}} n
\end{aligned}
$$

$$
\begin{aligned}
& +\frac{\sqrt{2}}{3 \sqrt{3}} n+\frac{\sqrt{53}}{5 \sqrt{30}} n+\frac{\sqrt{17}}{3 \sqrt{26}} n \\
& +\frac{\sqrt{53}}{6 \sqrt{21}} n-\frac{14 \sqrt{13}}{27} n-\frac{10 \sqrt{5}}{9} n \\
G A_{5}(B A(Y)[m, n])= & \frac{12 \sqrt{10}}{19} m n+\frac{3}{2} m n \\
& +\frac{10 \sqrt{14}}{39} n+\frac{\sqrt{91}}{10} n+\frac{6 \sqrt{38}}{37} n \\
& +\frac{4 \sqrt{3}}{7} n+\frac{4 \sqrt{114}}{43} n+\frac{4 \sqrt{133}}{47} n \\
& +\frac{12 \sqrt{2}}{17} n+\frac{2 \sqrt{42}}{13} n+\frac{10 \sqrt{26}}{51} n \\
& +\frac{15 \sqrt{3}}{26} n+\frac{2 \sqrt{30}}{11} n+\frac{6 \sqrt{78}}{53} n \\
& +\frac{12 \sqrt{21}}{55} n-\frac{60 \sqrt{10}}{19} n-5 n .
\end{aligned}
$$

Proof. Consider the boron- $\alpha$ nanotube $K=\mathrm{BA}(Y)[m, n]$. From Figure 6, we can see that there are eight partite subsets of edge set $E_{K}$ corresponding to their degree of end vertices which are given as

$$
\begin{aligned}
& E_{3,3}=\left\{p q \in E_{K} \mid d_{p}=d_{q}=3\right\}, \\
& E_{3,5}=\left\{p q \in E_{K} \mid d_{p}=3 \text { and } d_{q}=5\right\}, \\
& E_{3,6}=\left\{p q \in E_{K} \mid d_{p}=3 \text { and } d_{v}=6\right\}, \\
& E_{4,4}=\left\{p q \in E_{k} \mid d_{p}=d_{q}=4\right\}, \\
& E_{4,5}=\left\{p q \in E_{K} \mid d_{u}=4 \text { and } d_{v}=5\right\}, \\
& E_{4,6}=\left\{p q \in E_{K} \mid d_{p}=4 \text { and } d_{q}=6\right\}, \\
& E_{5,5}=\left\{p q \in E_{K} \mid d_{p}=d_{q}=5\right\}, \\
& E_{5,6}=\left\{p q \in E_{K} \mid d_{p}=5 \text { and } d_{v}=6\right\} .
\end{aligned}
$$

Therefore, we have $\left|E_{3,3}\right|=n / 2,\left|E_{3,5}\right|=n,\left|E_{3,6}\right|=n,\left|E_{4,4}\right|=$ $3 n / 2,\left|E_{4,5}\right|=2 n,\left|E_{4,6}\right|=n,\left|E_{5,5}\right|=n(3 m-8) / 2$, and $\left|E_{5,6}\right|=$ $n(2 m-5)$. From (2) and (4), the $A B C$ and $G A$ indices of $K$ are computed as 


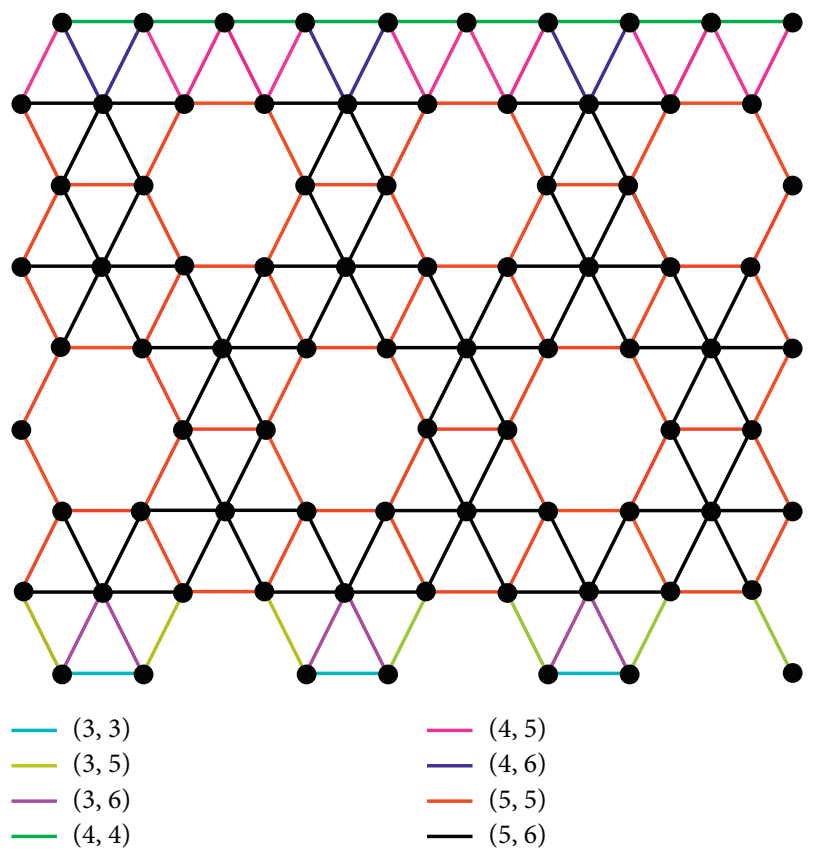

FIgURE 6: The edge partitions of $\mathrm{BA}(Y)[9,6]$ with respect to degree of end vertices.

$$
\begin{aligned}
& A B C(K)=\sum_{p q \in E_{3,3}} \sqrt{\frac{d_{p}+d_{q}-2}{d_{p} d_{q}}}+\sum_{p q \in E_{3,5}} \sqrt{\frac{d_{p}+d_{q}-2}{d_{p} d_{q}}} \\
& +\sum_{p q \in E_{3,6}} \sqrt{\frac{d_{p}+d_{q}-2}{d_{p} d_{q}}}+\sum_{p q \in E_{4,4}} \sqrt{\frac{d_{p}+d_{q}-2}{d_{p} d_{q}}} \\
& +\sum_{p q \in E_{4,5}} \sqrt{\frac{d_{p}+d_{q}-2}{d_{p} d_{q}}}+\sum_{p q \in E_{4,6}} \sqrt{\frac{d_{p}+d_{q}-2}{d_{p} d_{q}}} \\
& +\sum_{p q \in E_{5,5}} \sqrt{\frac{d_{p}+d_{q}-2}{d_{p} d_{q}}}+\sum_{p q \in E_{5,6}} \sqrt{\frac{d_{p}+d_{q}-2}{d_{p} d_{q}}} \\
& =\frac{6}{\sqrt{50}} m n+\frac{\sqrt{30}}{5} m n+\frac{3 \sqrt{6}}{8} n+\sqrt{\frac{2}{5}} n \\
& +\frac{\sqrt{7}}{3 \sqrt{2}} n+\frac{\sqrt{7}}{\sqrt{5}} n+\frac{1}{\sqrt{3}} n+\frac{1}{3} n-\frac{8 \sqrt{2}}{5} n-\frac{\sqrt{30}}{2} n, \\
& G A(K)=\sum_{p q \in E_{3,3}} \frac{2 \sqrt{d_{p} d_{q}}}{d_{p}+d_{q}}+\sum_{p q \in E_{3,5}} \frac{2 \sqrt{d_{p} d_{q}}}{d_{p}+d_{q}} \\
& +\sum_{p q \in E_{3,6}} \frac{2 \sqrt{d_{p} d_{q}}}{d_{p}+d_{q}}+\sum_{p q \in E_{4,4}} \frac{2 \sqrt{d_{p} d_{q}}}{d_{p}+d_{q}} \\
& +\sum_{p q \in E_{4,5}} \frac{2 \sqrt{d_{p} d_{q}}}{d_{p}+d_{q}}+\sum_{p q \in E_{4,6}} \frac{2 \sqrt{d_{p} d_{q}}}{d_{p}+d_{q}}
\end{aligned}
$$

$$
\begin{aligned}
& +\sum_{p q \in E_{5,5}} \frac{2 \sqrt{d_{p} d_{q}}}{d_{p}+d_{q}}+\sum_{p q \in E_{5,6}} \frac{2 \sqrt{d_{p} d_{q}}}{d_{p}+d_{q}} \\
& =\frac{4 \sqrt{30}}{11} m n+\frac{3}{2} m n+\frac{\sqrt{15}}{4} n+\frac{2 \sqrt{2}}{3} n \\
& +\frac{8 \sqrt{5}}{9} n+\frac{2 \sqrt{6}}{5} n-\frac{10 \sqrt{30}}{11} n-2 n .
\end{aligned}
$$

Similarly, there are nineteen edge partite subsets of $E_{K}$ corresponding to their degree sum of neighbors of end vertices, which are presented as

$$
\begin{aligned}
& S_{14,14}=\left\{p q \in E_{K} \mid \delta_{p}=\delta_{q}=14\right\}, \\
& S_{14,25}=\left\{p q \in E_{H} \mid \delta_{p}=14 \text { and } \delta_{q}=25\right\}, \\
& S_{14,26}=\left\{p q \in E_{K} \mid \delta_{p}=14 \text { and } \delta_{q}=26\right\}, \\
& S_{18,19}=\left\{p q \in E_{K} \mid \delta_{p}=18 \text { and } \delta_{q}=19\right\}, \\
& S_{18,24}=\left\{p q \in E_{K} \mid \delta_{p}=18 \text { and } \delta_{q}=24\right\}, \\
& S_{19,19}=\left\{p q \in E_{K} \mid \delta_{p}=\delta_{q}=19\right\}, \\
& S_{19,24}=\left\{p q \in E_{K} \mid \delta_{p}=19 \text { and } \delta_{q}=24\right\}, \\
& S_{26,27}=\left\{p q \in E_{K} \mid \delta_{p}=\delta_{q}=27\right\}, \\
& S_{19,28}=\left\{p q \in E_{K} \mid \delta_{p}=19 \text { and } \delta_{q}=28\right\}, \\
& S_{24,25,24}=\left\{p q \in E_{K} \mid \delta_{p}=\delta_{q}=24\right\}, \\
& S_{24,27}=\left\{p q \in E_{K} \mid \delta_{p}=24 \text { and } \delta_{q}=27\right\},
\end{aligned}
$$




$$
\begin{aligned}
& S_{27,28}=\left\{p q \in E_{K} \mid \delta_{p}=27 \text { and } \delta_{q}=28\right\}, \\
& S_{27,30}=\left\{p q \in E_{K} \mid \delta_{p}=27 \text { and } \delta_{q}=30\right\} .
\end{aligned}
$$

Therefore, we have $\left|S_{14,14}\right|=n / 2,\left|S_{14,25}\right|=n,\left|S_{14,26}\right|=n$, $\left|S_{18,19}\right|=n,\left|S_{18,24}\right|=n,\left|S_{19,19}\right|=n / 2,\left|S_{19,24}\right|=n,\left|S_{19,28}\right|=n$, $\left|S_{24,24}\right|=n / 2, \quad\left|S_{24,27}\right|=n, \quad\left|S_{24,28}\right|=n, \quad\left|S_{25,25}\right|=n / 2$, $\left|S_{25,26}\right|=n, \quad\left|S_{25,27}\right|=n, \quad\left|S_{25,30}\right|=n, \quad\left|S_{26,27}\right|=n, \quad\left|S_{27,27}\right|=$ $n(3 m-14) / 2, \quad\left|S_{27,28}\right|=n$, and $\left|S_{27,30}\right|=2 n(m-5)$. The representative edges of these partite sets corresponding to certain colors are presented in Figure 7. From (3) and (5), the fourth $A B C$ and fifth $G A$ indices of $\mathrm{K}$ are computed as

$$
\begin{aligned}
& A B C_{4}(K)=\sum_{p q \in S_{14,14}} \sqrt{\frac{\delta_{p}+\delta_{q}-2}{\delta_{p} \delta_{q}}}+\sum_{p q \in S_{14,25}} \sqrt{\frac{\delta_{p}+\delta_{q}-2}{\delta_{p} \delta_{q}}} \\
& +\sum_{p q \in S_{14,26}} \sqrt{\frac{\delta_{p}+\delta_{q}-2}{\delta_{p} \delta_{q}}}+\sum_{p q \in S_{18,19}} \sqrt{\frac{\delta_{p}+\delta_{q}-2}{\delta_{p} \delta_{q}}} \\
& +\sum_{p q \in S_{18,24}} \sqrt{\frac{\delta_{p}+\delta_{q}-2}{\delta_{p} \delta_{q}}}+\sum_{p q \in S_{19,19}} \sqrt{\frac{\delta_{p}+\delta_{q}-2}{\delta_{p} \delta_{q}}} \\
& +\sum_{p q \in S_{19,24}} \sqrt{\frac{\delta_{p}+\delta_{q}-2}{\delta_{p} \delta_{q}}}+\sum_{p q \in S_{19,28}} \sqrt{\frac{\delta_{p}+\delta_{q}-2}{\delta_{p} \delta_{q}}} \\
& +\sum_{p q \in S_{24,24}} \sqrt{\frac{\delta_{p}+\delta_{q}-2}{\delta_{p} \delta_{q}}}+\sum_{p q \in S_{24,27}} \sqrt{\frac{\delta_{p}+\delta_{q}-2}{\delta_{p} \delta_{q}}} \\
& +\sum_{p q \in S_{24,28}} \sqrt{\frac{\delta_{p}+\delta_{q}-2}{\delta_{p} \delta_{q}}}+\sum_{p q \in S_{25,25}} \sqrt{\frac{\delta_{p}+\delta_{q}-2}{\delta_{p} \delta_{q}}} \\
& +\sum_{p q \in S_{25,26}} \sqrt{\frac{\delta_{p}+\delta_{q}-2}{\delta_{p} \delta_{q}}}+\sum_{p q \in S_{25,27}} \sqrt{\frac{\delta_{p}+\delta_{q}-2}{\delta_{p} \delta_{q}}} \\
& +\sum_{p q \in S_{25,30}} \sqrt{\frac{\delta_{p}+\delta_{q}-2}{\delta_{p} \delta_{q}}}+\sum_{p q \in S_{26,27}} \sqrt{\frac{\delta_{p}+\delta_{q}-2}{\delta_{p} \delta_{q}}} \\
& +\sum_{p q \in S_{27,27}} \sqrt{\frac{\delta_{p}+\delta_{q}-2}{\delta_{p} \delta_{q}}}+\sum_{p q \in S_{27,28}} \sqrt{\frac{\delta_{p}+\delta_{q}-2}{\delta_{p} \delta_{q}}} \\
& +\sum_{p q \in S_{27,30}} \sqrt{\frac{\delta_{p}+\delta_{q}-2}{\delta_{p} \delta_{q}}} \\
& =\frac{\sqrt{13}}{9} m n+\frac{2 \sqrt{5}}{9} m n+\frac{\sqrt{26}}{28} n+\frac{\sqrt{37}}{5 \sqrt{14}} n+\frac{\sqrt{38}}{2 \sqrt{91}} n
\end{aligned}
$$

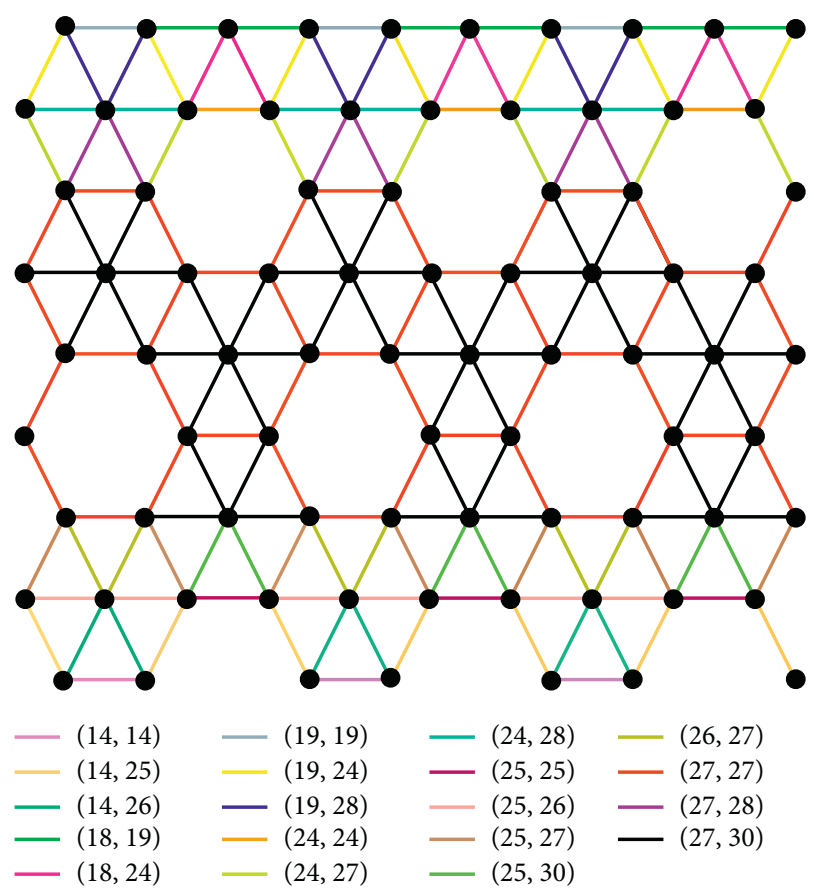

Figure 7: The edge partitions of $\mathrm{BA}(X)[9,6]$ with respect to degree sum of neighbor vertices.

$$
\begin{aligned}
+ & \frac{\sqrt{35}}{3 \sqrt{38}} n+\frac{\sqrt{10}}{6 \sqrt{3}} n+\frac{3}{19} n+\frac{\sqrt{41}}{2 \sqrt{114}} n+\frac{3 \sqrt{5}}{2 \sqrt{133}} n \\
+ & \frac{\sqrt{46}}{48} n+\frac{7}{18 \sqrt{2}} n+\frac{5}{4 \sqrt{21}} n+\frac{2 \sqrt{3}}{25} n \\
& +\frac{7}{5 \sqrt{26}} n+\frac{\sqrt{2}}{3 \sqrt{3}} n+\frac{\sqrt{53}}{5 \sqrt{30}} n+\frac{\sqrt{17}}{3 \sqrt{26}} n \\
& +\frac{\sqrt{53}}{6 \sqrt{21}} n-\frac{14 \sqrt{13}}{27} n-\frac{10 \sqrt{5}}{9} n, \\
G A_{5}(K)= & \sum_{p q \in S_{14,14}} \frac{2 \sqrt{\delta_{p} \delta_{q}}}{\delta_{p}+\delta_{q}}+\sum_{p q \in S_{14,25}} \frac{2 \sqrt{\delta_{p} \delta_{q}}}{\delta_{p}+\delta_{q}} \\
& +\sum_{p q \in S_{14,26}} \frac{2 \sqrt{\delta_{p} \delta_{q}}}{\delta_{p}+\delta_{q}}+\sum_{p q \in S_{18,19}} \frac{2 \sqrt{\delta_{p} \delta_{q}}}{\delta_{p}+\delta_{q}} \\
& +\sum_{p q \in S_{18,24}} \frac{2 \sqrt{\delta_{p} \delta_{q}}}{\delta_{p}+\delta_{q}}+\sum_{p q \in S_{19,19}} \frac{2 \sqrt{\delta_{p} \delta_{q}}}{\delta_{p}+\delta_{q}} \\
& +\sum_{p q \in S_{19,24}} \frac{2 \sqrt{\delta_{p} \delta_{q}}}{\delta_{p}+\delta_{q}}+\sum_{p q \in S_{19,23}} \frac{2 \sqrt{\delta_{p} \delta_{q}}}{\delta_{p}+\delta_{q}}
\end{aligned}
$$


TABLE 2: Degree-based topological indices of boron nanotubes.

\begin{tabular}{lccr}
\hline Index & $\mathrm{BT}[m, n]$ & $\mathrm{BA}(X)[m, n]$ & $\mathrm{BA}(Y)[m, n]$ \\
\hline$A B C$ & $(2.37171 m-1.02333) n$ & $(1.943973 m-0.190827) n$ & $(1.943973 m-0.732831) n$ \\
$A B C_{4}$ & $(1.0458 m-0.10448) n$ & $(0.921774 m+0.645374) n$ & $(0.897521 m+0.023558) n$ \\
$G A$ & $(4.5 m-3.12122) n$ & $(3.491718 m-1.040331) n$ & $(3.491718 m-2.100829) n$ \\
$G A_{5}$ & $(4.5 m-3.17234) n$ & $(3.497228 m-1.067682) n$ & $(2.498614 m+2.874247) n$ \\
\hline
\end{tabular}

$$
\begin{aligned}
& +\sum_{p q \in S_{24,24}} \frac{2 \sqrt{\delta_{p} \delta_{q}}}{\delta_{p}+\delta_{q}}+\sum_{p q \in S_{24,27}} \frac{2 \sqrt{\delta_{p} \delta_{q}}}{\delta_{p}+\delta_{q}} \\
& +\sum_{p q \in S_{24,28}} \frac{2 \sqrt{\delta_{p} \delta_{q}}}{\delta_{p}+\delta_{q}}+\sum_{p q \in S_{25,25}} \frac{2 \sqrt{\delta_{p} \delta_{q}}}{\delta_{p}+\delta_{q}} \\
& +\sum_{p q \in S_{25,26}} \frac{2 \sqrt{\delta_{p} \delta_{q}}}{\delta_{p}+\delta_{q}}+\sum_{p q \in S_{25,27}} \frac{2 \sqrt{\delta_{p} \delta_{q}}}{\delta_{p}+\delta_{q}} \\
& +\sum_{p q \in S_{25,30}} \frac{2 \sqrt{\delta_{p} \delta_{q}}}{\delta_{p}+\delta_{q}}+\sum_{p q \in S_{26,27}} \frac{2 \sqrt{\delta_{p} \delta_{q}}}{\delta_{p}+\delta_{q}} \\
& +\sum_{p q \in S_{27,27}} \frac{2 \sqrt{\delta_{p} \delta_{q}}}{\delta_{p}+\delta_{q}}+\sum_{p q \in S_{27,28}} \frac{2 \sqrt{\delta_{p} \delta_{q}}}{\delta_{p}+\delta_{q}} \\
& +\sum_{p q \in S_{27,30}} \frac{2 \sqrt{\delta_{p} \delta_{q}}}{\delta_{p}+\delta_{q}} \\
& =\frac{12 \sqrt{10}}{19} m n+\frac{3}{2} m n+\frac{10 \sqrt{14}}{39} n+\frac{\sqrt{91}}{10} n+\frac{6 \sqrt{38}}{37} n \\
& +\frac{4 \sqrt{3}}{7} n+\frac{4 \sqrt{114}}{43} n+\frac{4 \sqrt{133}}{47} n+\frac{12 \sqrt{2}}{17} n \\
& +\frac{2 \sqrt{42}}{13} n+\frac{10 \sqrt{26}}{51} n+\frac{15 \sqrt{3}}{26} n+\frac{2 \sqrt{30}}{11} n \\
& +\frac{6 \sqrt{78}}{53} n+\frac{12 \sqrt{21}}{55} n-\frac{60 \sqrt{10}}{19} n-5 n .
\end{aligned}
$$

\section{Conclusion}

In this paper, we study important classes of boron nanotubes and formulated $A B C$, the fourth $A B C, G A$ and the fifth $G A$ indices of their molecular graphs by using the edge partition technique. The simplified expressions of these indices for boron triangular and boron- $\alpha$ nanotubes are given in Table 2. These results can be used in detecting some physical and chemical properties of these boron nanotubes, and in this sense, these results will be helpful in structural chemistry.

\section{Conflicts of Interest}

The authors declare that they have no conflicts of interest.

\section{Acknowledgments}

This research is supported by Higher Education Commission, Pakistan, via Grant no. 5331/Federal/NRPU/R\&D/ HEC/2016.

\section{References}

[1] S. J. Cyvin and I. Gutman, Lecture Notes in Chemistry, Vol. 46, Springer-Verlag, Berlin, Germany, 1988.

[2] N. Trinajstić and I. Gutman, "Mathematical chemistry," Croatica Chemica Acta, vol. 75, pp. 329-356, 2002.

[3] N. Trinajstić, Chemical Graph Theory, CRC Press, Boca Raton, FL, USA, 1992.

[4] I. Gutman and O. E. Polansky, Mathematical Concepts in Organic Chemistry, Springer-Verlag, New York, NY, USA, 1986.

[5] Z. Slanina, F. Uhlk, S.-L. Lee, and E. Osawa, "Geometrical and thermodynamic approaches to the relative stabilities of fullerene isomers," MATCH Communications in Mathematical and in Computer Chemistry, vol. 44, pp. 335-348, 2001.

[6] D. Vukicevic, F. Cataldo, O. Ori, and A. Graovac, "Topological efficiency of $C_{66}$ fullerene," Chemical Physics Letters, vol. 501, pp. 442-445, 2011.

[7] F. Cataldo, A. Graovac, and O. Ori, The Mathematics and Topology of Fullerenes, Springer, Dordrecht, Netherlands, 2011.

[8] I. Nadeem and H. Shaker, "On eccentric connectivity index of $\mathrm{TiO}_{2}$ nanotubes," Acta Chimica Slovenica, vol. 63-62, pp. 363-368, 2016.

[9] M. M. Zobair, A. M. Malik, H. Shaker, and N. Rehman, "Eccentricity based topological invariants of triangulane dendrimers," Utilitas Mathematica, vol. 107, pp. 193-206, 2018.

[10] J. B. Liu, X. F. Pan, F. T. Hu, and F. F. Hu, "Asymptotic Laplacian-energy-like invariant of lattices," Applied Mathematics and Computation, vol. 253, pp. 205-214, 2015.

[11] J. B. Liu, X. F. Pan, L. Yu, and D. Li, "Complete characterization of bicyclic graphs with minimal Kirchhoff index," Discrete Applied Mathematics, vol. 200, pp. 95-107, 2016.

[12] M. Randić, "Characterization of molecular branching," Journal of the American Chemical Society, vol. 97, pp. 66096615, 1975.

[13] I. Gutman and B. Furtula, Recent Results in the Theory of Randić Index, University of Kragujevac, Kragujevac, Serbia, 2008.

[14] X. Li and I. Gutman, Mathematical Aspects of Randić-Type Molecular Structure Descriptors, University of Kragujevac, Kragujevac, Serbia, 2006. 
[15] E. Estrada, L. Torres, L. Rodriguez, and I. Gutman, “An atombond connectivity index: modelling the enthalpy of formation of alkanes," Indian Journal of Chemistry, vol. 37A, pp. 849855, 1998.

[16] E. Estrada, "Atom-bond connectivity and the energetic of branched alkanes," Chemical Physics Letters, vol. 463, pp. 422-425, 2008.

[17] I. Gutman, J. Tošović, S. Radenković, and S. Marković, "On atom-bond connectivity index and its chemical applicability," Indian Journal of Chemistry, vol. 51A, pp. 690-694, 2012.

[18] M. Ghorbani and M. A. Hosseinzadeh, "Computing $\mathrm{ABC}_{4}$ index of nanostar dendrimers," Optoelectronics and Advanced Materials: Rapid Communications, vol. 4, no. 9, pp. 14191422, 2010.

[19] D. Vukičević and B. Furtula, "Topological index based on the ratios of geometrical and arithmetical means of end-vertex degrees of edges," Journal of Mathematical Chemistry, vol. 46, pp. 1369-1376, 2009.

[20] K. C. Das, "On geometric-arithmetic index of graphs," MATCH Communications in Mathematical and in Computer Chemistry, vol. 64, pp. 619-630, 2010.

[21] K. C. Das, I. Gutman, and B. Furtula, "Survey on geometricarithmetic indices of graphs," MATCH Communications in Mathematical and in Computer Chemistry, vol. 65, pp. 595644, 2011.

[22] A. Graovac, M. Ghorbani, and M. A. Hosseinzadeh, "Computing fifth geometric-arithmetic index for nanostar dendrimers," Journal of Mathematical Nanoscience, vol. 1, no. 1, pp. 33-42, 2011.

[23] J. Kunstmann and A. Quandt, "Broad boron sheets and boron nanotubes: an ab initio study of structural, electronic and mechanical properties," Physical Review B, vol. 74, pp. 354$362,2006$.

[24] S. Battersby, Boron Nanotubes Could Outperform Carbon, New Scientist, London, UK, January 2008, http://www. newscientist.com.

[25] P. Miller, Boron Nanotubes Beat Carbon at Its Own Game, Engadget, San Francisco, CA, USA, January 2008, http://www. engadget.com.

[26] Y. Chen, Nanotechnology in SpaceNanowerk, June 2007, http://www.nanowerk.com.

[27] M.-L. Sun, Z. Slanina, and S.-L. Lee, "Square/hexagon route towards the boron-nitrogen clusters," Chemical Physics Letters, vol. 233, pp. 279-283, 1995.

[28] Z. Slanina, M.-L. Sun, and S.-L. Lee, "AM1 stability prediction: $\mathrm{B}^{36} \mathrm{~N}^{24}>\mathrm{B}^{36} \mathrm{P}^{24}>\mathrm{Al}^{36} \mathrm{~N}^{24}>\mathrm{Al}^{36} \mathrm{P}^{24}$, Journal of Molecular Structure (Theochem), vol. 334, pp. 229-233, 1995.

[29] Z. Slanina, M.-L. Sun, and S.-L Lee, "Computations of boron and boron-nitrogen cages," Nanostructured Materials, vol. 8, pp. 623-635, 1997.

[30] H. T. Pham, L. V. Duong, N. M. Tam, M. P. Pham-Ho, and M. T. Nguyen, "The boron conundrum: bonding in the bowl $\mathrm{B}^{30}$ and $\mathrm{B}^{36}$, fullerene $\mathrm{B}^{40}$ and triple ring $\mathrm{B}^{42}$ clusters," Chemical Physics Letters, vol. 608, pp. 295-302, 2014.

[31] R. K. F. Lee, B. J. Cox, and J. M. Hill, "Ideal polyhedral model for boron nanotubes with distinct bond lengths," Journal of Physical Chemistry C, vol. 113, pp. 19794-19805, 2009.

[32] H. Tang and S. Ismail-Beigi, "Novel precursors for boron nanotubes: the competition of two-center and three-center bonding in boron sheets," Physical Review Letters, vol. 99, pp. 115501-115504, 2007.

[33] A. K. Singh, A. Sadrzadeh, and B. I. Yakobson, "Probing properties of boron $\alpha$-tubes by ab initio calculations," Nano Letters, vol. 8, no. 5, pp. 1314-1317, 2008.
[34] X. Yang, Y. Ding, and J. Ni, "Ab initio prediction of stable boron sheets and boron nanotubes: structure, stability and electronic properties," Physics Review B, vol. 77, p. 041402(R), 2008.

[35] P. Manuel, "Computational aspects of carbon and boron nanotubes," Molecules, vol. 15, no. 12, pp. 8709-8722, 2010. 


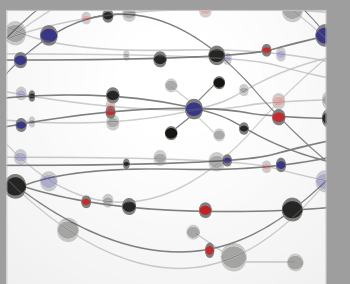

The Scientific World Journal
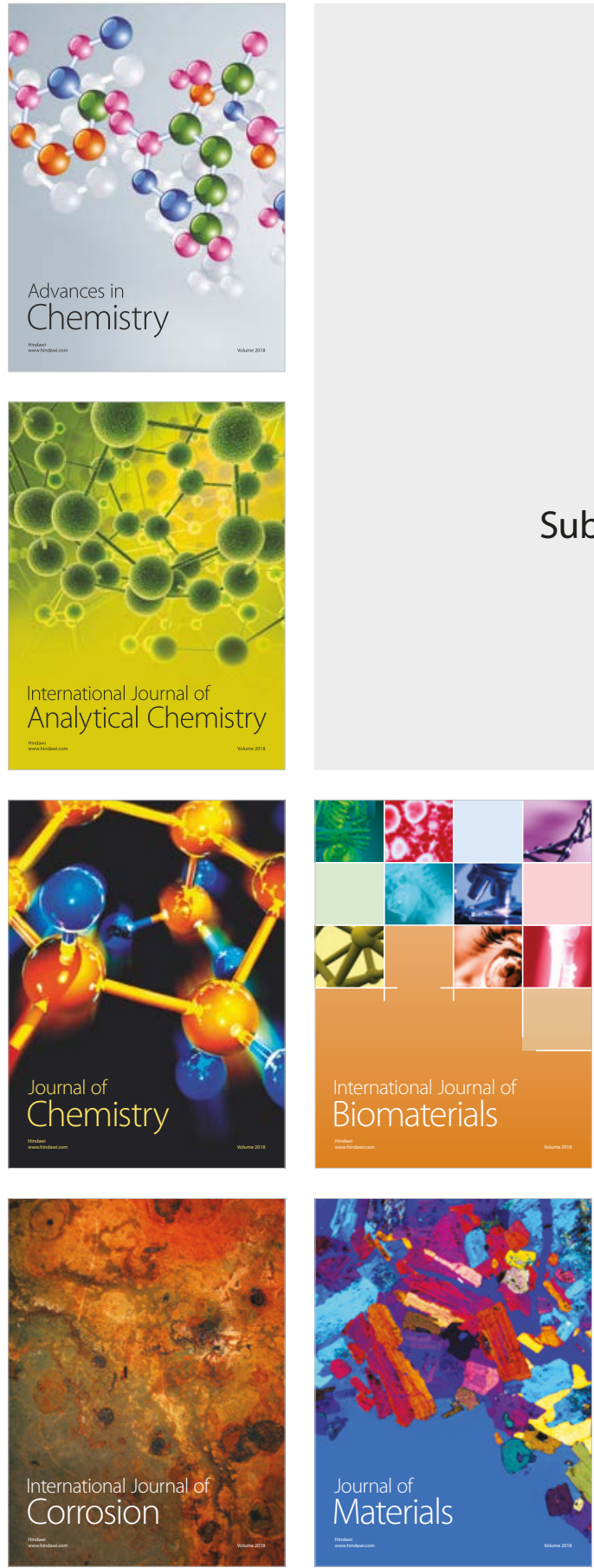

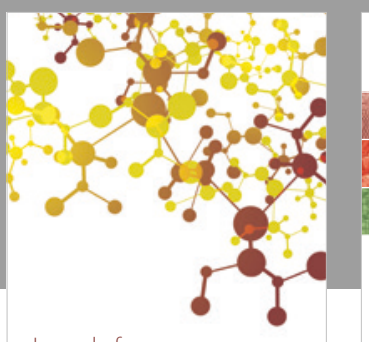

Journal of

Applied Chemistry
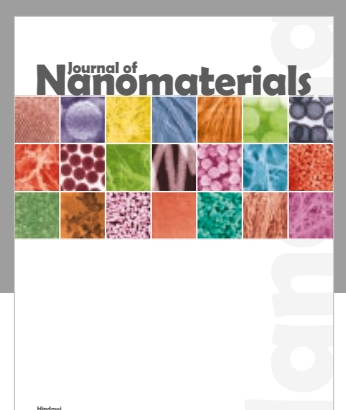

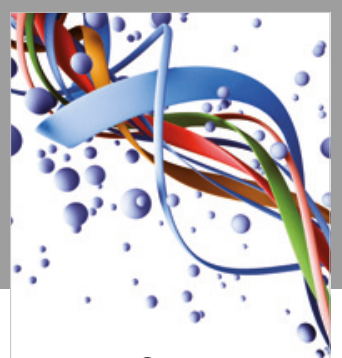

Scientifica

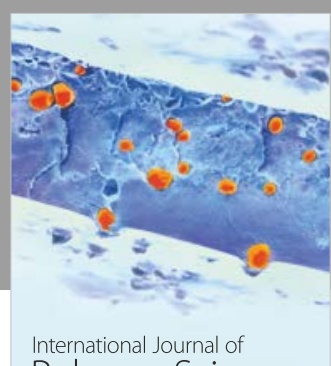

Polymer Science

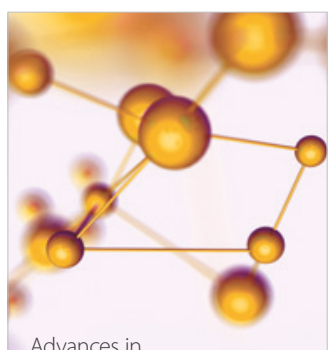

Physical Chemistry
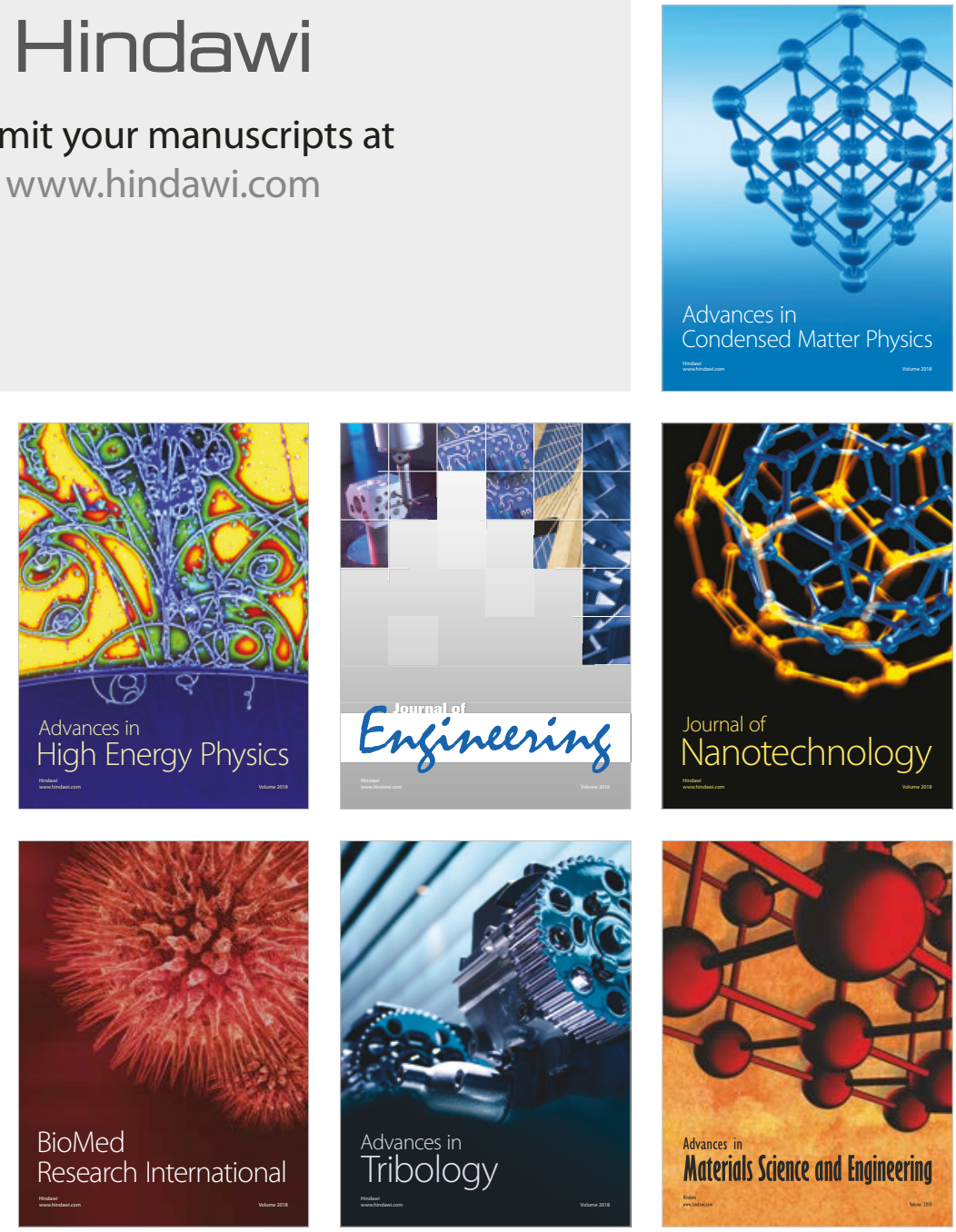